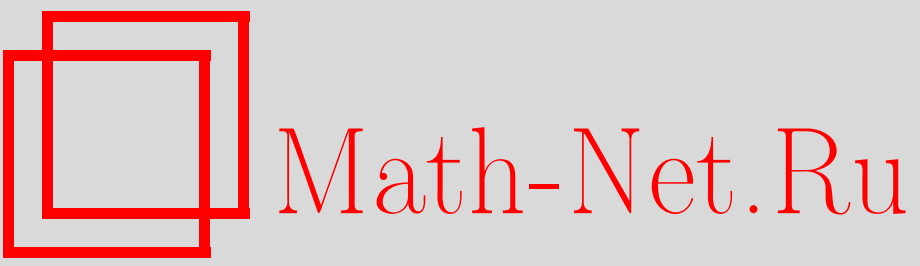

В. В. Кварацхелия, В. И. Тариеладзе, Диагонально канонические гауссовские случайные элементы, Теория вероятн. и ее примен., 2013, том 58, выпуск 2, 282297

DOI: https://doi.org/10.4213/tvp4507

Использование Общероссийского математического портала Math-Net.Ru подразумевает, что вы прочитали и согласны с пользовательским соглашением http://www.mathnet.ru/rus/agreement

Параметры загрузки:

IP: 3.89 .185 .249

26 апреля 2023 г., $14: 43: 30$ 
(С) 2013 г. КВАРАЦХЕЛИЯ В. В. ${ }^{*}$, ТАРИЕЛАДЗЕ В. И.*

\section{ДИАГОНАЛЬНО КАНОНИЧЕСКИЕ ГАУССОВСКИЕ СЛУЧАЙНЫЕ ЭЛЕМЕНТЫ ${ }^{1)}$}

Посвящается 80-летию академика Н. Н. Вахания

Гауссовский случайный элемент $\eta$ со значениями в банаховом пространстве $X$ с базисом Шаудера $\mathbf{e}=\left(e_{n}\right)$ назовем диагонально каноническим (для краткости $D$-каноническим) относительно базиса е, если распределение $\eta$ совпадает с распределением случайного элемента вида $B \xi$, где $\xi$ - гауссовский случайный элемент со значениями в $X$, компоненты которого относительно базиса е стохастически независимы, и $B: X \rightarrow X$ - линейный непрерывный оператор. В данной статье мы доказываем, что если $X=l_{p}$, $1 \leqslant p<\infty$ и $p \neq 2$, или $X=c_{0}$, то существует гауссовский случайный элемент $\eta$ в $X$, которьй не является $D$-каноническим относительно естественного базиса $X$. Мы выводим этот результат в случае $X=l_{p}, 2<p<\infty$, или $X=c_{0}$ из следующего утверждения, аналог которого ранее был известен для некоторых банаховых пространств, не обладающих безусловным базисом Шаудера: если $X=l_{p}, 2<p<\infty$, или $X=c_{0}$, то существует гауссовский случайный элемент $\eta$ в $X$ такой, что распределение $\eta$ не совпадает с распределением суммь почти наверное сходящегося ряда $\sum_{n=1}^{\infty} x_{n} g_{n}$ в $X$, где $\left(x_{n}\right)$ - безусловно суммируемая последовательность элементов в $X u\left(g_{n}\right)$ - последовательность стохастически независимых стандартных гауссовских случайных величин.

Ключевые слова и фразы: диагонально канонический гауссовский случайный элемент, безусловно канонический гауссовский случайный элемент, гауссовский ковариационный оператор, котип банаховых пространств, $r$-ядерный оператор, суммирующий оператор, гауссовское среднее свойство, $g l_{2}$-банаховы пространства.

1. Введение. Поводом для написания данной работы стал следующий вопрос проф. Н. Н. Вахания, заданный авторам этой статьи.

Вопрос 1.1. Пусть $X-$ одно из пространств $l_{p}, 1 \leqslant p<\infty$, или $c_{0}$, и пусть $\eta$ - гауссовский случайный элемент со значениями

* Институт вычислительной математики им. Н.И. Мусхелишвили Грузинского технического университета, Тбилиси, Грузия; v_kvaratskhelia@yahoo.com; vajatarieladze@yahoo.com

1) Работа выполнена при поддержке Национального научного фонда им. Шота Руставели (грант GNSF/ST09_99_3-104). 
в X. Найдутся ли гауссовский случайный элемент $\xi$ со значениями в $X$, имеюший стохастически независимье компоненты, и линейное непрерывное отображение $B: X \rightarrow X$ такие, что $B \xi=\eta$ п.н. или $B \xi$ имеет то же самое распределение, что и $\eta$ ?

Мы даем отрицательный ответ на данный вопрос в случаях, когда $X=l_{p}, 1 \leqslant p<\infty, p \neq 2$, и $X=c_{0}$. Наше доказательство основано на результатах и понятиях, которые имеют самостоятельный интерес. Среди них - описание гауссовских распределений в пространствах $l_{p}$, $1 \leqslant p<\infty[1],[2]$, ковариационный оператор [3]-[5] и другие важные разработки Н.Н. Вахания.

Во втором пункте приведены основные понятия, нужные для дальнейшего изложения, введено понятие диагонально канонического гауссовского случайного элемента в банаховом пространстве с базисом Шаудера и рассмотрены случаи, когда ответ на вопрос 1.1 положителен.

В п. 3 доказывается, что не все гауссовские случайные элементы в пространстве $l_{p}, 1 \leqslant p<2$, являются диагонально каноническими относительно естественного базиса (теорема 3.2 ).

В п. 4 мы сначала вводим понятие безусловно канонического гауссовского случайного элемента в произвольном банаховом пространстве (определение 4.1), затем доказываем, что в банаховом пространстве с безусловным базисом каждый диагонально канонический гауссовский случайный элемент является безусловно каноническим (предложение 4.1(c)), и, наконец, показываем, что не все гауссовские случайные элементы в $l_{p}, 2<p<\infty$, являются безусловно (и, следовательно, диагонально) каноническими (теорема 4.5).

Пункт 5 содержит доказательство утверждения, что не все гауссовские случайные элементы в $c_{0}$ являются безусловно (и, следовательно, диагонально) каноническими (теорема 5.2).

2. D-канонические случайные элементы. Мы рассматриваем только действительные векторные пространства и центрированные гауссовские случайные элементы. Норму в банаховом пространстве $X$ обозначим через $\|\cdot\|$. Для банахова пространства $X$ сопряженное пространство $X^{*}$ снабжено дуальной нормой. Значение функционала $x^{*} \in X^{*}$ на $x \in X$ обозначим через $\left\langle x, x^{*}\right\rangle$. Скалярное произведение в гильбертовом пространстве обозначим через $(\cdot \mid \cdot)$. Для базиса Шаудера $\mathbf{e}=\left(e_{n}\right)$ банахова пространства $X$ обозначим через $\mathbf{e}^{*}=\left(e_{n}^{*}\right)$ его биортогональную последовательность в $X^{*}$.

Говорят, что случайный элемент $\eta$ со значениями в банаховом пространстве $X$ имеет конечный ранг, если существует конечномерное векторное подпространство $X_{0}$ пространства $X$ такое, что $\eta \in X_{0}$ почти наверное (п.н.).

Пусть $X$ - банахово пространство с базисом Шаудера е. Мы бу- 
дем говорить, что гауссовский случайный элемент $\eta$ со значениями в $X$ является $D$-каноническим относительно базиса е, если распределение $\eta$ совпадает с распределением случайного элемента вида $B \xi$, где $\xi-$ гауссовский случайный элемент в $X$, для которого е-компоненты $\left\langle\xi, e_{n}^{*}\right\rangle$, $n=1,2, \ldots$, являются стохастически независимыми, и $B: X \rightarrow X$ есть линейное непрерывное отображение.

Предложение 2.1. Пусть $X-$ бесконечномерное банахово пространство с базисом Шаудера $\mathbf{e}=\left(e_{n}\right)$ и $\eta-$ чентрированный гауссовский случайньий элемент конечного ранга в $X$. Тогда $\eta$ является D-каноническим относительно базиса е.

Д о к а з а т е л ь с т в о. Зафиксируем наименьшее (относительно теоретико-множественного включения $\subset$ ) конечномерное векторное подпространство $X_{0}$ пространства $X$ такое, что $\eta \in X_{0}$ п.н.

Если $X_{0}=\{0\}$, то $\eta$ очевидно является $D$-каноническим относительно базиса е.

Если $\operatorname{dim} X_{0}=1$, то для некоторых $0 \neq x_{1} \in X_{0}$ и стандартной гауссовской случайной величины $g_{1}$ имеем $\eta=x_{1} g_{1}$ п.н. Положим $\xi=$ $e_{1} g_{1}$ и рассмотрим отображение $B: X \rightarrow X$, определенное равенством

$$
B x=\left\langle x, e_{1}^{*}\right\rangle x_{1}, \quad x \in X .
$$

Тогда $B \xi=\eta$ п.н. и, следовательно, $\eta$ является $D$-каноническим относительно базиса е.

Пусть теперь $\operatorname{dim} X_{0}=n \geqslant 2$. Тогда $\eta=\sum_{k=1}^{n} x_{k} g_{k}$ п.н. для некоторых $x_{1}, \ldots, x_{n}$ из $X_{0}$ и независимых стандартных гауссовских случайных величин $g_{1}, \ldots, g_{n}$. Положим $\xi=\sum_{k=1}^{n} e_{k} g_{k}$ и рассмотрим отображение $B: X \rightarrow X$, определенное равенством

$$
B x=\sum_{k=1}^{n}\left\langle x, e_{k}^{*}\right\rangle x_{k}, \quad x \in X .
$$

Тогда $B \xi=\eta$ п.н. Следовательно, и в этом случае $\eta$ является $D$-каноническим относительно базиса е. Предложение 2.1 доказано.

Мы опускаем несложное доказательство следующего предложения.

Предложение 2.2. Пусть $X-$ бесконечномерное банахово пространство с базисом Шаудера $\left(e_{n}\right)$ и $\eta$ - чентрированный гауссовский случайный элемент в $X$, который является $D$-каноническим относительно базиса $\left(e_{n}\right)$. Тогда:

(а) если $t_{n}>0, n=1,2, \ldots$, то $\eta$ является D-каноническим относительно базиса $\left(t_{n} e_{n}\right)$;

(b) если $\left(e_{n}^{\prime}\right)$ - базис Шаудера пространства $X$, который эквивалентен базису $\left(e_{n}\right)$, то $\eta$ является D-каноническим относительно базиса $\left(e_{n}^{\prime}\right)$. 
Рассмотрим теперь случай гильбертова пространства.

Предложение 2.3. Пусть $X-$ произвольное бесконечномерное гильбертово пространство с ортонормированным базисом $\mathbf{e}=\left(e_{n}\right) u$ пусть $\eta$ - чентрированный гауссовский случайный элемент со значениями в $X$. Тогда $\eta$ является $D$-каноническим относительно базиса е.

Д о к а з а т е ль с т в о. Согласно предложению 2.1, мы можем предположить, что $\eta$ не является случайным элементом конечного ранга. Тогда хорошо известно, что $\eta=\sum_{k=1}^{\infty} x_{k} g_{k}$ п.н. для некоторой последовательности $x_{1}, \ldots, x_{n}, \ldots$, составленной из ненулевых попарно ортогональных элементов из $X$ таких, что $\sum_{k=1}^{\infty}\left\|x_{k}\right\|^{2}<\infty$, и для некоторых стохастически независимых стандартных гауссовских случайных величин $g_{1}, \ldots, g_{n}, \ldots$ Положим $\xi=\sum_{k=1}^{\infty}\left\|x_{k}\right\| e_{k} g_{k}$ и рассмотрим отображение $B: X \rightarrow X$, определенное равенством

$$
B x=\sum_{k=1}^{\infty}\left(x \mid e_{k}\right) \frac{x_{k}}{\left\|x_{k}\right\|}, \quad x \in X .
$$

Заметим, что $B$ является линейной изометрией, поскольку $\left(x_{k} /\left\|x_{k}\right\|\right)$ есть ортонормированная последовательность в $X$. Тогда $B \xi=\eta$ п.н., и, следовательно, $\eta$ является $D$-каноническим относительно базиса е. Предложение 2.3 доказано.

Следствие 2.1. Пусть $X-$ произвольное бесконечномерное гильбертово пространство с безусловньлм базисом $\mathbf{e}=\left(e_{n}\right)$ и пусть $\eta-$ чентрированный гауссовский случайный элемент со значениями в $X$. Тогда $\eta$ является D-каноническим относительно базиса $\mathbf{e .}$

Д о к а з а т е л ь с т в о. По предложению 2.2(a) можно считать, что безусловный базис $\left(e_{n}\right)$ является нормированным. Известно, что произвольный нормированный безусловный базис гильбертова пространства эквивалентен произвольному ортонормированному базису (см., например, $[8$, с. 71$])$. Поэтому данное следствие вытекает из предложения 2.3 и предложения $2.2(\mathrm{~b})$.

3 а м е ч а н и е 2.1. Нам неизвестно, верно ли заключение следствия 2.1 , если $\left(e_{n}\right)$ - произвольный (т.е. не обязательно безусловный) базис гильбертова пространства $X$.

3. $D$-канонические случайные элементы в пространствах $l_{p}$, $1 \leqslant p<2$. Пусть $X-$ банахово пространство; говорят, что оператор $R: X^{*} \rightarrow X$ является:

- ковариационным оператором центрированного гауссовского случайного элемента $\xi$ в $X$, если

$$
\left\langle R x_{1}^{*}, x_{2}^{*}\right\rangle=\mathbf{E}\left\langle\xi, x_{1}^{*}\right\rangle\left\langle\xi, x_{2}^{*}\right\rangle, \quad x_{1}^{*}, x_{2}^{*} \in X^{*}
$$


- гауссовской ковариацией, если существует гауссовский случайный элемент $\xi$ в $X$ с ковариационным оператором $R$.

Ясно, что если $X$ - банахово пространство и $R: X^{*} \rightarrow X$ - гауссовская ковариация, то $R$ симметричный (т.е. $\left\langle R x_{1}^{*}, x_{2}^{*}\right\rangle=\left\langle R x_{2}^{*}, x_{1}^{*}\right\rangle$ для всех $\left.x_{1}^{*}, x_{2}^{*} \in X^{*}\right)$ и положительный (т.е. $\left\langle R x^{*}, x^{*}\right\rangle \geqslant 0$ для каждого $x^{*} \in X^{*}$ ) оператор.

Мы будем существенно использовать следующий результат.

Теорема 3.1 (см. [1], [2]). Пусть $1 \leqslant p<\infty, X=l_{p} u \mathbf{e}=$ $\left(e_{n}\right)$ - естественньии базис пространства $X$. В этом случае оператор $R: X^{*} \rightarrow X$ является гауссовской ковариацией тогда и только тогда, когда он симметричный, положительньй и

$$
\sum_{n=1}^{\infty}\left\langle R e_{n}^{*}, e_{n}^{*}\right\rangle^{p / 2}<\infty
$$

Прежде чем сформулировать одно следствие теоремы 3.1 , напомним следующее определение.

Пусть $Y, Z$ - банаховы пространства, $0<r \leqslant 1$. Линейный непрерывный оператор $S: Y \rightarrow Z$ называется $r$-ядерныл, если существуют последовательности $y_{n}^{*} \in Y^{*}, z_{n} \in Z, n=1,2, \ldots$, такие, что

$$
\sum_{n=1}^{\infty}\left\|y_{n}^{*}\right\|^{r}\left\|z_{n}\right\|^{r}<\infty, \quad S y=\sum_{n=1}^{\infty}\left\langle y, y_{n}^{*}\right\rangle z_{n}, \quad y \in Y .
$$

Следствие 3.1. Пусть $1 \leqslant p \leqslant 2, X=l_{p}$, e $=\left(e_{n}\right)$ - естественньй базис пространства $X$, и пусть $\eta$ - D-канонический относительно базиса е гауссовский случайньй элемент со значениями в $X$. Тогда ковариачионный оператор $R_{\eta}$ случайного элемента $\eta$ является р/2-ядернылм.

Д о к а з а т е л ь с т в о. По определению, распределение $\eta$ совпадает с распределением случайного элемента вида $B \xi$, где $\xi$ есть гауссовский случайный элемент в $X$, у которого е-компоненты $\left\langle\xi, e_{n}^{*}\right\rangle, n=$ $1,2, \ldots$, являются стохастически независимыми, и $B: X \rightarrow X-$ линейное непрерывное отображение.

Так как $\xi=\sum_{n=1}^{\infty}\left\langle\xi, e_{n}^{*}\right\rangle e_{n}$, ясно, что

$$
B \xi=\sum_{n=1}^{\infty}\left\langle\xi, e_{n}^{*}\right\rangle B e_{n}
$$

Поскольку гауссовские случайные величины $\left\langle\xi, e_{n}^{*}\right\rangle, n=1,2, \ldots$, центрированы и стохастически независимы, то из этого равенства получим

$$
R_{\eta} x^{*}=R_{B \xi} x^{*}=\sum_{n=1}^{\infty} \sigma_{n}^{2}\left\langle B e_{n}, x^{*}\right\rangle B e_{n}, \quad x^{*} \in X^{*},
$$


где

$$
\sigma_{n}=\left(\mathbf{E}\left\langle\xi, e_{n}^{*}\right\rangle^{2}\right)^{1 / 2}=\left\langle R e_{n}^{*}, e_{n}^{*}\right\rangle^{1 / 2}, \quad n=1,2, \ldots
$$

Так как $\xi$ есть гауссовский случайный элемент со значениями в $X$, то по теореме 3.1 имеем

$$
\sum_{n=1}^{\infty}\left\|\sigma_{n}^{2} B e_{n}\right\|^{p / 2}\left\|B e_{n}\right\|^{p / 2} \leqslant\|B\|^{p} \sum_{n=1}^{\infty} \sigma_{n}^{p}=\|B\|^{p} \sum_{n=1}^{\infty}\left\langle R e_{n}^{*}, e_{n}^{*}\right\rangle^{p / 2}<\infty .
$$

Следовательно, $R_{\eta}$ есть $p / 2$-ядерный оператор. Следствие 3.1 доказано.

Теперь применим следствие 3.1 для доказательства результата, сформулированного в аннотации данной работы, в случае $X=l_{p}$, $1 \leqslant p<2$.

Теорема 3.2. Пусть $1 \leqslant p<2, X=l_{p} u \mathbf{e}=\left(e_{n}\right)$ - естественный базис пространства $X$. Тогда существует гауссовский случайный элемент $\eta$ со значениями в $X$, который не является D-каноническим относительно базиса е.

Д о к а з а т е л с с в о. Отдельно рассмотрим два случая.

Случай 1: $1<p<2$. Согласно [6, следствие 9(ii)], для каждого $0<r<1$ существует гауссовский случайный элемент $\eta$ в $X$, ковариационный оператор которого не является $r$-ядерным. В частности, в $X$ существует гауссовский случайный элемент $\eta$, ковариационный оператор которого не является $p / 2$-ядерным; согласно следствию 3.1 , такой случайный элемент $\eta$ не может быть $D$-каноническим относительно базиса е.

Cлучай 2: $p=1$. Согласно [7, теорема 1], в пространстве $X=l_{1}$ существует гауссовский случайный элемент $\eta$, ковариационный оператор которого не является 1/2-ядерным; согласно следствию 3.1, такой случайный элемент $\eta$ не может быть $D$-каноническим относительно базиса е.

Следствие 3.2. (а) Если $X=l_{1} u\left(e_{n}\right)$ - произвольный безусловньии базис пространства $X$, то существует чентрированньй гауссовский случайньй элемент $\eta$ в $X$, который не является $D$-каноническим относительно базиса $\left(e_{n}\right)$.

(b) Если $1<p<2, X=l_{p} u\left(e_{n}\right)$ - произвольньй симметрический базис пространства $X$, то существует иентрированньии гауссовский случайный элемент $\eta$ в $X$, который не является D-каноническим относительно базиса $\left(e_{n}\right)$.

Д о к а з а т е л ь с т в о. (а) По предложению 2.2(a) можно считать, что безусловный базис $\left(e_{n}\right)$ является нормированным. Известно, что произвольный нормированный безусловный базис пространства $X$ эквивалентен каноническому базису пространства $X$ (см. [8, с. 71]). 
Поэтому данное утверждение вытекает из теоремы 3.2 и предложения $2.2(\mathrm{~b})$.

(b) По предложению 2.2(a) можно считать, что симметрический базис $\left(e_{n}\right)$ является нормированным. Известно, что произвольный нормированный симметрический базис пространства $X$ эквивалентен каноническому базису пространства $X$ (см. $[8$, с. 129]). Поэтому, данное утверждение вытекает из теоремы 3.2 и предложения 2.2(b).

3 а м е ч а н и е 3.1. Мы не знаем ответа на следующий вопрос относительно следствия 3.1. Пусть $1 \leqslant p<2, X=l_{p}$, e $=\left(e_{n}\right)-$ естественньий базис пространства $X$, и пусть $\eta$ - гауссовский случайный элемент со значениями в $X$ такой, что ковариационный оператор $R_{\eta}$ случайного элемента $\eta$ является $p / 2$-ядерным. Будет ли $\eta$ $D$-каноническим относительно базиса $\mathbf{e}$ ?

3 а м е ч а н и е 3.2. Следствие 3.2(b) оставляет открытым следующий вопрос. Пусть $1<p<2, X=l_{p}$, и пусть $\eta-$ произвольньий центрированный гауссовский случайный элемент со значениями в $X$. Существует ли безусловньй базис в пространстве $X$, относительно которого $\eta$ является $D$-каноническим?

4. $U$ - и $D$-канонические случайные элементы в пространствах $l_{p}, 2<p<\infty$. Метод доказательства теоремы 3.2 не проходит в случае $2<p<\infty$. В этом пункте мы разрабатываем способы, необходимые для получения отрицательного ответа на вопрос 1.1 в случае $2<p<\infty$.

Следуя [9], мы скажем, что последовательность $\left(x_{n}\right)$ элементов банахова пространства $X$ является безусловно суммируемой, если ряд $\sum_{n=1}^{\infty} x_{n}$ сходится безусловно в $X$.

Зафиксируем последовательность $\left(g_{n}\right)$ стохастически независимых стандартных гауссовских случайных величин, заданных на вероятностном пространстве $(\Omega, \mathscr{A}, \mathbf{P})$.

Теорема 4.1 (см. [10]). Для банахова пространства $X$ следующие утверждения эквивалентнь:

(i) для каждой безусловно суммируемой последовательности $\left(x_{n}\right)$ элементов пространства $X$ ряд $\sum_{n=1}^{\infty} x_{n} g_{n}$ сходится п.н. в $X$;

(ii) $X$ имеет конечный котип;

(iii) для каждой безусловно суммируемой последовательности $\left(x_{n}\right)$ элементов пространства $X$ существует $\Omega_{0} \in \mathscr{A}, \mathbf{P}\left(\Omega_{0}\right)=1$, такое, что для всех $\omega \in \Omega_{0}$ ряд $\sum_{n=1}^{\infty} x_{n} g_{n}(\omega)$ сходится безусловно в $X$.

Для произвольной суммируемой последовательности $\left(x_{n}\right)$ в банаховом пространстве $X$ ряд $\sum_{n=1}^{\infty} x_{n} g_{n}$ может не сходиться п.н. в $X$. Однако в общем случае верно следующее утверждение.

Теорема 4.2 (см. [11]). Пусть $\left(x_{n}\right)$ - безусловно суммируемая последовательность элементов банахова пространства $X$, для кото- 
рой п.н. сходится ряд $\sum_{n=1}^{\infty} x_{n} g_{n}$ в $X$. Тогда существует $\Omega_{0} \in \mathscr{A}$, $\mathbf{P}\left(\Omega_{0}\right)=1$, такое, ито для всех $\omega \in \Omega_{0}$ ряд $\sum_{n=1}^{\infty} x_{n} g_{n}(\omega)$ cходится безусловно в $X$.

Этими результатами мотивируется следующее определение.

О п р е д е л е н и е 4.1. Гауссовский случайный элемент $\xi$ со значениями в банаховом пространстве $X$ назовем безусловно каноническим (для краткости $U$-каноническим), если существуют последовательность $\left(x_{n}\right)$ элементов пространства $X$ и последовательность $\left(g_{n}\right)$ стохастически независимых стандартных гауссовских случайных величин такие, что:

- ряд $\sum_{n=1}^{\infty} x_{n}$ сходится безусловно в $X$;

- ряд $\sum_{n=1}^{\infty} x_{n} g_{n}$ сходится п.н. в $X$;

- распределение $\xi$ совпадает с распределением суммы $\sum_{n=1}^{\infty} x_{n} g_{n}$.

Имеется следующая связь между безусловно каноническими и диагонально каноническими гауссовскими случайными элементами.

Предложение 4.1. Пусть $X$ - банахово пространство с безусловныл базисом Шаудера $\mathbf{e}=\left(e_{n}\right)$.

(а) $\left[15\right.$, лемма 2.1]. Если $R: X^{*} \rightarrow X$ гауссовская ковариаиия, то ряд $\sum_{n=1}^{\infty}\left\langle R e_{n}^{*}, e_{n}^{*}\right\rangle^{1 / 2} e_{n}$ сходится в $X$.

(b) Если $\xi$ - гауссовский случайный элемент со значениями в $X$, для которого $\left\langle\xi, e_{n}^{*}\right\rangle, n=1,2, \ldots$, - чентрированные и стохастически независимье случайные величинь, то $\xi$ есть $U$-канонический гауссовский случайный элемент.

(c) Если $\eta$ есть D-канонический относительно базиса е гауссовский случайный элемент в $X$, то $\eta$ есть $U$-канонический гауссовский случайньй элемент.

Д о к а з а т е ль с т в о. (b) Рассмотрим случайный элемент $\xi$, удовлетворяющий требованиям условия (b). Обозначим:

$$
\sigma_{n}=\left(\mathbf{E}\left\langle\xi, e_{n}^{*}\right\rangle^{2}\right)^{1 / 2}=\left\langle R e_{n}^{*}, e_{n}^{*}\right\rangle^{1 / 2}, \quad x_{n}=\sigma_{n} e_{n}, \quad n=1,2, \ldots .
$$

Зафиксируем последовательность $\left(g_{n}\right)$ стохастически независимых стандартных гауссовских случайных величин.

Имеем:

- согласно (а), ряд $\sum_{n=1}^{\infty} x_{n}$ сходится безусловно в $X$;

- ряд $\sum_{n=1}^{\infty} x_{n} g_{n}$ сходится п.н. в $X$, так как ряд $\sum_{n=1}^{\infty}\left\langle\xi, e_{n}^{*}\right\rangle e_{n}$ сходится к $\xi$ в пространстве $X$ и для каждого $n \in \mathbf{N}$ случайные элементы $\left\langle\xi, e_{n}^{*}\right\rangle e_{n}$ и $x_{n} g_{n}$ имеют одинаковые распределения;

- распределение $\xi$ совпадает с распределением суммы $\sum_{n=1}^{\infty} x_{n} g_{n}$ по той же причине, что и в предыдущем случае.

(c) По определению, распределение $\eta$ совпадает с распределением случайного элемента, имеющего вид $B \xi$, где $\xi$ - гауссовский случайный элемент в $X$, чьи е-компоненты $\left\langle\xi, e_{n}^{*}\right\rangle, n=1,2, \ldots$, стохастически 
независимы, и $B: X \rightarrow X$ есть линейное непрерывное отображение. Согласно (b), $\xi$ есть $U$-канонический гауссовский случайный элемент в $X$. Отсюда очевидно вытекает, что $B \xi$ также является $U$-каноническим гауссовским случайным элементом в $X$.

Предложение 4.1 доказано.

Для полноты изложения мы приводим доказательство следующего известного результата.

Теорема 4.3. В банаховом пространстве котипа 2 каждый гауссовский случайный элемент является $U$-каноническим.

Д о к а з а т е ль с т в о. Пусть $X$ - банахово пространство котипа 2 и $\eta$ - гауссовский случайный элемент в $X$. Согласно $[15$, теорема 4.1], существует гауссовский случайный элемент $\xi$ в $l_{2}$ и линейное непрерывное отображение $U: l_{2} \rightarrow X$ такие, что распределение $U \xi$ совпадает с распределением $\eta$. По предложению $2.3, \xi$ является $D$ каноническим относительно естественного базиса в $l_{2}$. Поэтому, по предложению $4.1(\mathrm{c}), \xi$ является $U$-каноническим. Таким образом, $U \xi$ (и, следовательно, $\eta$ ) является $U$-каноническим.

3 а м е ч а н и е 4.1. Следующий вопрос, связанный с предложением 4.1(c), имеет отрицательный ответ. Пусть $X-$ банахово пространство с безусловныл базисом Шаудера $\mathbf{e}=\left(e_{n}\right)$ и $\eta$ есть $U$ канонический гауссовский случайный әлемент в X. Является ли $\eta$ диагонально каноническим гауссовским случайным элементом относительно базиса $\mathbf{e}$ ?

В самом деле, пусть $1 \leqslant p<2$ и $X=l_{p}$. Тогда по теореме 4.3 , каждый гауссовский случайный элемент в $X$ является $U$-каноническим, но по теореме 3.2 не каждый гауссовский случайный элемент в $X$ является диагонально каноническим относительно естественного базиса пространства $X$.

Заметим, что п.н. непрерывная реализация винеровского процесса $\left(W_{t}\right)_{t \in[0,1]}$ индуцирует гауссовский случайный элемент в $X=C([0,1])$, который является безусловно каноническим [11].

Относительно отрицательных утверждений до сих пор был известен только следующий результат.

Теорема 4.4 (см. [12, с. 61, предложение]). Существуют сепарабельное банахово пространство $E$ и гауссовский случайный элемент $\eta$ со значениями в $E$ такие, что $\eta$ не является $U$-каноническим.

3 а м е ч а н и е 4.2. В [12, с. 61-64] доказано, что в банаховом пространстве $E=K\left(l_{2}\right)$ всех компактных операторов в $l_{2}$ существует гауссовский случайный элемент, который не является $U$-каноническим. Пространство $K\left(l_{2}\right)$ имеет базис Шаудера, но не обладает безусловным базисом Шаудера и не имеет конечного котипа. 
Имея в виду теорему 4.1 и замечание 4.2, можно было бы предположить, что в произвольном банаховом пространстве с безусловным базисом и конечным котипом каждый гауссовский случайный элемент является $U$-каноническим (см. [12, замечание 3 , с. 63]). Следующее утверждение показывает, что это предположение неверно.

Теорема 4.5. Пусть $X=l_{p}, 2<p<\infty, u \mathbf{e}=\left(e_{n}\right)$ есть безусловный базис пространства $X$. Тогда:

(а) существует гауссовский случайньй элемент со значениями в $X$, который не является $U$-каноническим;

(b) существует гауссовский случайньй элемент со значениями в $X$, который не является $D$-каноническим относительно базиса е.

Из предложения 4.1(c) вытекает, что теорема 4.5(b) есть следствие теоремы 4.5(a).

Остальная часть данного пункта посвящается доказательству теоремы 4.5(a).

Предложение 4.2. Пусть $X$ - банахово пространство, $E \subset$ $X$ - измеримое по Борелю векторное подпространство, $\left(x_{n}\right)-$ последовательность элементов в $X u\left(\zeta_{n}\right)$ - последовательность стохастически независимьх симметрически распределенных действительных случайных величин таких, что:

1) $\mathbf{P}\left[\zeta_{n}=0\right]<1, n=1,2, \ldots$

2) ряд $\sum_{n=1}^{\infty} x_{n} \zeta_{n}$ сходится п.н. в $X$,

3) $\sum_{n=1}^{\infty} x_{n} \zeta_{n} \in E$ n.н.

Тогда $x_{n} \in E$ для всех $n=1,2, \ldots$.

Д о к а з а т е л ь с т в о. Покажем, например, что $x_{1} \in E$. Для остальных индексов доказательство аналогично.

Так как $\left(\zeta_{n}\right)$ есть последовательность стохастически независимых симметрически распределенных действительных случайных величин, то последовательности $\left(\zeta_{1}, \zeta_{2}, \ldots, \zeta_{n}, \ldots\right)$ и $\left(\zeta_{1},-\zeta_{2}, \ldots,-\zeta_{n}, \ldots\right)$ распределены одинаково. Отсюда следует, что случайные элементы $x_{1} \zeta_{1}+$ $\sum_{n=2}^{\infty} x_{n} \zeta_{n}$ и $x_{1} \zeta_{1}-\sum_{n=2}^{\infty} x_{n} \zeta_{n}$ распределены одинаково. Следовательно, согласно условию 3$), x_{1} \zeta_{1}-\sum_{n=2}^{\infty} x_{n} \zeta_{n} \in E$ п.н. Теперь из соотношений $x_{1} \zeta_{1}+\sum_{n=2}^{\infty} x_{n} \zeta_{n} \in E$ и $x_{1} \zeta_{1}-\sum_{n=2}^{\infty} x_{n} \zeta_{n} \in E$ получим $2 x_{1} \zeta_{1} \in E$ п.н. Из этого и из условия 1$)$, поскольку $E$ есть векторное подпространство, вытекает, что $x_{1} \in E$.

Следствие 4.1. Пусть $X-$ банахово пространство, $E \subset X-$ замкнутое векторное подпространство и $\xi$ есть $U$-канонический гауссовский случайный элемент со значениями в $X$ такой, что $\xi \in E$ п.н. Тогда $\xi$ есть $U$-канонический гауссовский случайньй элемент со значениями в $E$.

Д о к а з а т е л ь с т в о. Так как $\xi$ есть $U$-канонический гауссовский случайный элемент в $X$, то найдутся последовательность $\left(x_{n}\right)$ эле- 
ментов в $X$ и последовательность $\left(g_{n}\right)$ стохастически независимых стандартных гауссовских случайных величин такие, что для них выполнены условия определения 4.1. Поскольку $\xi \in E$ п.н. и распределение $\xi$ совпадает с распределением суммы $\sum_{n=1}^{\infty} x_{n} g_{n}$, имеем $\sum_{n=1}^{\infty} x_{n} g_{n} \in E$ п.н. По предложению $4.2, x_{n} \in E, n=1,2, \ldots$. Отсюда, поскольку $E-$ замкнутое векторное подпространство пространства $X$, получим, что ряд $\sum_{n=1}^{\infty} x_{n}$ сходится безусловно в $E$ и ряд $\sum_{n=1}^{\infty} x_{n} g_{n}$ сходится п.н. в $E$. Следовательно, $\xi$ является $U$-каноническим гауссовским случайным элементом в $E$.

Следствие 4.2. Пусть $X$ - банахово пространство такое, ито каждый гауссовский случайный элемент со значениями в $X$ является $U$-каноническим, и пусть $E \subset X$ - замкнутое векторное подпространство. Тогда каждый гауссовский случайный элемент со значениями в Е является $U$-каноническим.

Д о к а з а т е л ь с т в о. Пусть $\xi$ - гауссовский случайный элемент со значениями в $E$. Тогда $\xi$ есть гауссовский случайный элемент со значениями также и в $X$. По предположению, $\xi$ как гауссовский случайный элемент со значениями в $X$ является $U$-каноническим. Тогда по следствию 4.1 можно заключить, что $\xi$ является $U$-каноническим и как гауссовский случайный элемент со значениями в $E$. Следствие 4.2 доказано.

Пусть $Y_{1}, Y_{2}, Y_{3}$ - банаховы пространства. Линейный непрерывный оператор $T: Y_{1} \rightarrow Y_{3}$ называется $Y_{2}$ - бакторизуемьлм, если существуют линейные непрерывные операторы $T_{1}: Y_{1} \rightarrow Y_{2}$ и $T_{2}: Y_{2} \rightarrow Y_{3}$ такие, что $T=T_{2} T_{1}$.

Лемма 4.1. Пусть $X$ - банахово пространство, $\left(x_{n}\right)$ - безусловно суммируемая последовательность элементов пространства $X$ и $T: l_{2} \rightarrow X-$ такой линейньй непрерыьвный оператор, что

$$
T T^{*} x^{*}=\sum_{n=1}^{\infty}\left\langle x_{n}, x^{*}\right\rangle x_{n}, \quad x^{*} \in X^{*} .
$$

Тогда T есть с $c_{0}$-Факторизуемьй оператор.

Д о к а з а т е л ь с т в о. Легко проверить, что равенство

$$
S h=\sum_{n=1}^{\infty}\left(h \mid e_{k}\right) x_{k}, \quad h \in l_{2},
$$

определяет линейный непрерывный оператор $S: l_{2} \rightarrow X$ такой, что $S S^{*}=T T^{*}$. Из последнего равенства имеем

$$
\left\|T^{*} x^{*}\right\|^{2}=\left\|S^{*} x^{*}\right\|^{2}, \quad x^{*} \in X^{*} .
$$


Определим отображение $V_{0}: S^{*}\left(X^{*}\right) \rightarrow T^{*}\left(X^{*}\right)$ с помощью равенства

$$
V_{0} S^{*} x^{*}=T^{*} x^{*}, \quad x^{*} \in X^{*} .
$$

Из (4.1) легко следует, что $V_{0}$ - корректно определенная линейная изометрия из $S^{*}\left(X^{*}\right)$ в $T^{*}\left(X^{*}\right)$. Пусть $V: l_{2} \rightarrow l_{2}$ - линейное непрерывное продолжение $V_{0}$. Ясно, что $V S^{*}=T^{*}$ и, следовательно,

$$
T=S V^{*} .
$$

Заметим теперь, что $S$ есть $c_{0}$-факторизуемый оператор. В самом деле, имеем $S=S_{1} S_{2}$, где $S_{2}$ есть естественное вложение $l_{2}$ в $c_{0}$ и $S_{1}: c_{0} \rightarrow X$ определен равенством

$$
S_{1} x=\sum_{n=1}^{\infty}\left\langle x, e_{n}^{*}\right\rangle x_{n}, \quad x \in c_{0},
$$

где $\left(e_{n}^{*}\right)$ - биортогональная последовательность естественного базиса $\left(e_{n}\right)$ пространства $c_{0}$. Из равенств $S=S_{1} S_{2}$ и (4.2) получим $T=T_{1} T_{2}$, где $T_{1}=S_{1}$ и $T_{2}=S_{2} V^{*}$. Следовательно, $T$ есть $c_{0}$-факторизуемый оператор. Лемма 4.1 доказана.

Непосредственным следствием леммы 4.1 является следующее утверждение.

Предложение 4.3. Пусть $X-$ банахово пространство и $T: l_{2} \rightarrow$ $X$ - линейный непрерывный оператор такой, что TT* есть ковариаиионный оператор $U$-канонического гауссовского случайного элемента $\xi$ со значениями в $X$. Тогда $T$ есть $c_{0}$-факторизуемьй оператор.

Д о к а з а т е л ь с т в о. Так как $\xi$ есть $U$-канонический гауссовский случайный элемент, то существуют безусловно суммируемая последовательность $\left(x_{n}\right)$ в $X$ и последовательность стохастически независимых стандартных гауссовских случайных величин $\left(g_{n}\right)$ такие, что распределение $\xi$ совпадает с распределением суммы $\sum_{n=1}^{\infty} x_{n} g_{n}$. Следовательно, ковариационный оператор $T T^{*}$ случайного элемента $\xi$ можно представить в следующем виде

$$
T T^{*} x^{*}=\sum_{n=1}^{\infty}\left\langle x_{n}, x^{*}\right\rangle x_{n}, \quad x^{*} \in X^{*} .
$$

Тогда согласно лемме 4.1 можно заключить, что оператор $T$ является $c_{0}$-факторизуемым. Предложение 4.3 доказано.

В дальнейшем нам понадобятся следующие определения.

О п р е д е л е н и е 4.2. Пусть $Y, Z$ - банаховы пространства. Линейный оператор $T: Y \rightarrow Z$ называется (абсолютно) суммирующим, если для каждой безусловно суммируемой последовательности $\left(y_{n}\right)$ элементов пространства $Y$ имеем $\sum_{n=1}^{\infty}\left\|T y_{n}\right\|<\infty$. 
О п р е д е л е н и е 4.3. Банахово пространство $Y$ называется:

- $g l_{2}$-банаховым пространством, если для каждого суммирующего оператора $S: Y \rightarrow l_{2}$ существует пространство с положительной мерой $(\Omega, \mathscr{A}, \nu)$ такое, что $S$ является $L_{1}(\Omega, \mathscr{A}, \nu)$-факторизуемым;

- наследственно $g l_{2}$-пространством, если каждое замкнутое векторное подпространство пространства $Y$ есть $g l_{2}$-пространство.

Нетрудно убедиться в том, что каждое банахово пространство с безусловным базисом Шаудера является $g l_{2}$-пространством. А. Гротендиком была высказана гипотеза, что все банаховы пространства суть $g l_{2}$-пространства. Первый пример пространства, не являющегося $g l_{2}$ банаховым, был построен Гордоном и Льюисом [13]. Более подробную информацию о $g l_{2}$-пространствах можно найти в [9].

О п р е д е л е н и е 4.4 (см. [14, определение 1.1]). Говорят, что банахово пространство $Y$ обладает гауссовским средним свойством (для краткости GAP), если для каждого линейного непрерывного оператора $T: l_{2} \rightarrow Y$, для которого $T^{*}$ является суммирующим, оператор $T T^{*}$ есть гауссовская ковариация.

Следующее утверждение показывает, что GAP является наследственным свойством.

Предложение 4.4 (см. [14, с. 564]). Пусть $Y$ - банахово пространство, имеющее GAP, $и X$ - замкнутое векторное подпространство пространства $Y$. Тогда $X$ также имеет GAP.

Д о к а з а т е л ь с т в о. Зафиксируем линейный непрерывный оператор $T: l_{2} \rightarrow X$, для которого $T^{*}$ есть суммирующий оператор. Пусть $J: X \rightarrow Y$ - естественное вложение и $S=J T: l_{2} \rightarrow Y$. Так как $T^{*}$ - суммирующий оператор, то $S^{*}=T^{*} J^{*}$ также является суммирующим. Поскольку $Y$ имеет GAP, то оператор $S S^{*}$ является гауссовской ковариацией. Пусть $\left(e_{n}\right)$ - естественный базис пространства $l_{2}$ и $\left(g_{n}\right)$ - последовательность стохастически независимых стандартных гауссовских случайных величин. Из того, что $S S^{*}$ есть гауссовская ковариация, стандартным путем можно вывести, что ряд $\sum_{n} S e_{n} g_{n}$ сходится п.н. в $Y$. Ясно, что $S e_{n} g_{n}=T e_{n} g_{n} \in X, n=1,2, \ldots$ Так как $X$ - замкнутое векторное подпространство пространства $Y$, то ряд $\sum_{n} T e_{n} g_{n}$ сходится п.н. в $X$. Очевидно, что случайный элемент $\xi$ в $X$, который п.н. равен сумме ряда $\sum_{n} T e_{n} g_{n}$, является гауссовским случайным элементом с ковариационным оператором $T T^{*}$. Следовательно, мы показали, что для любого линейного непрерывного оператора $T: l_{2} \rightarrow X$, для которого $T^{*}$ есть суммирующий оператор, $T T^{*}$ является гауссовской ковариацией. Таким образом, $X$ имеет GAP. Предложение 4.4 доказано.

Предложение 4.5 (см. [15, лемма 2.2 и теорема 2.1]). Пусть $Y-$ банахово пространство конечного котипа и с безусловным базисом Шаудера. Тогда $Y$ имеет GAP. 
Следующее утверждение является ключевым моментом в доказательстве теоремы 4.5(a).

Предложение 4.6. Пусть рефлексивное банахово пространство $Y$ с безусловным базисом Шаудера имеет конечный котип и пусть каждый гауссовский случайный элемент в $Y$ является $U$-каноническим. Тогда:

(a) $Y$ является наследственно $\mathrm{gl}_{2}$-банаховыл пространством;

(b) $Y$ имеет котип $q$ для всех $q>2$.

Д о к а з а т е л ь с т о. (а) Зафиксируем замкнутое векторное подпространство $X$ пространства $Y$. Известно, что $X$ является $g l_{2}$ банаховым пространством тогда и только тогда, когда $X^{*}$ является $g l_{2}$-банаховым пространством [9, предложение 17.9]. Теперь покажем, что $X^{*}$ является $g l_{2}$-банаховым пространством. Рассмотрим произвольный суммирующий оператор $S: X^{*} \rightarrow l_{2}$. Так как $Y-$ банахово пространство с конечным котипом и безусловным базисом, то по предложению 4.5 оно имеет GAP. Тогда, согласно предложению $4.4, X$ также имеет GAP. Следовательно, $S^{*} S$ есть ковариационный оператор гауссовского случайного элемента $\xi$ со значениями в $X$. По предположению, $\xi$ является $U$-каноническим в $Y$. Тогда, согласно следствию $4.1, \xi$ является $U$-каноническим как гауссовский случайный элемент со значениями в $X$. Таким образом, получаем, что $S^{*} S$ есть ковариационный оператор $U$-канонического гауссовского случайного элемента со значениями в $X$. Отсюда, по предложению 4.3 , получаем, что $S^{*}: l_{2} \rightarrow X^{* *}=X$ является $c_{0}$-факторизуемым. Следовательно, $S$ является $l_{1}$-факторизуемым и утверждение (a) доказано.

(b) Согласно [16, лемма 1], из (а) следует, что $Y$ имеет слабый котип 2 в смысле [17]. Следовательно, согласно [18, предложение 10.7(2)] $Y$ имеет котип $q$ для всех $q>2$.

3 а м е ч а н и е 4.3. Известно, что каждое банахово пространство котипа 2 с безусловным базисом Шаудера является наследственно $g l_{2}$ банаховым пространством; однако неизвестно, каждое ли банахово пространство котипа 2 является наследственно $g l_{2}$-пространством (см. $[14$, проблема 1.15]). Мы не знаем, можно ли в предположениях предложения 4.6 утверждать, что $Y$ является пространством котипа 2.

Д ок аз а т ел ь с т в о т е о р е м ы 4.5. (а) Так как пространство $l_{p}$ имеет безусловный базис, то оно является $g l_{2}$-банаховым. Кроме того, $l_{p}$ имеет котип $p$ и не имеет котипа $q<p$. Следовательно, по предложению 4.6(b), существует гауссовский случайный элемент со значениями в $l_{p}$, который не является $U$-каноническим.

(b) следует из (a) согласно предложению 4.1(c).

5. $D$-канонические случайные элементы в пространстве $c_{0}$. Нам понадобится следующий результат. 
Теорема 5.1 (см. [19, теорема 19]). Пусть X-сепарабельное банахово пространство и $\eta$ - гауссовский случайный элемент со значениями в $X$. Тогда существуют замкнутое векторное подпространство $E \subset c_{0}$, гауссовский случайный элемент $\xi$ со значениями в $E$ u линейный непрерьвный оператор $U: E \rightarrow X$ такие, что распределение

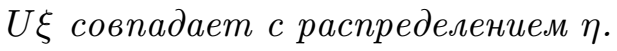

Теперь мы можем доказать модификацию теоремы 4.5 для случая $X=c_{0}$.

Теорема 5.2. Пусть $X=c_{0} u \mathbf{e}=\left(e_{n}\right)$ - естественный базис пространства $X$. Тогда:

(а) существует гауссовский случайный әлемент со значениями в $X$, который не является $U$-каноническим;

(b) существует гауссовский случайный элемент со значениями в $X$, который не является $D$-каноническим относительно базиса $\mathbf{e}$.

Д о к а з а т е л ь с т в о. (а) Допустим, что каждый гауссовский случайный элемент со значениями в $X$ является $U$-каноническим. Отсюда, согласно следствию 4.2 , можно заключить, что если $E-$ замкнутое векторное подпространство пространства $c_{0}$, то каждый гауссовский случайный элемент со значениями в $E$ является также и $U$ каноническим. По теореме 5.1 отсюда следует, что каждый гауссовский случайный элемент в каждом сепарабельном банаховом пространстве является $U$-каноническим (так как очевидно, что линейный непрерывный образ $U$-канонического случайного элемента есть $U$-канонический случайный элемент). Но это противоречит теореме 4.4 (или теореме $4.5(\mathrm{a}))$.

(b) следует из (а) согласно предложению 4.1(c), так как естественный базис $\mathbf{e}=\left(e_{n}\right)$ пространства $X$ есть безусловный базис.

В заключение выражаем признательность рецензенту за внимательное прочтение работы и критические замечания, большинство которых учтены. В частности, благодаря им в тексте появились предложение 2.2 , следствие 2.1 , замечание 2.1 , следствие 3.2 и замечание 3.2 .

\section{СПИСОК ЛИТЕРАТУРЫ}

1. Вахания H. H. О нормальных распределениях в пространстве $l_{p}$. - Теория вероятн. и ее примен., 1964, т. 9, в. 4, с. 737-738.

2. Vakhania N.N. Sur les répartitions de probabilités dans les espaces de suites numériques. - C. R. Acad. Sci. Paris, 1965, v. 260, № 5, p. 1560-1562.

3. Вахания H. Н. Ковариационный оператор вероятностного распределения в пространстве Банаха. - Сообщ. АН ГССР, 1968, т. 51, № 1, с. 35-40.

4. Vakhania N. N. Probability Distributions in Linear Spaces. New York-Oxford: NorthHolland, 1981, 123 p.

5. Vakhaniya N. N., Tarieladze V.I., Chobanyan S.A. Probability Distributions on Banach Spaces. Dordrecht: Reidel, 1987, 482 p. 
6. Kühn T., Linde $W$. The $r$-nuclearity $(0<r \leq 1)$ of Gaussian covariances. - Math. Nachr., 1980, v. 95, p. 165-175.

7. Kühn T. On the $r$-nuclearity of Gaussian covariances and the composition of nuclear operators. - Math. Ann., 1983, v. 262, p. 377-381.

8. Lindenstrauss J., Tzafriri L. Classical Banach Spaces. I: Sequence Spaces. BerlinHeidelberg-New York: Springer-Verlag, 1977, 190 p.

9. Diestel J., Jarchow H., Tonge A. Absolutely summing operators. Cambridge: Cambridge Univ. Press, 1995, 474 p.

10. Kvaratskhelia V.V. On unconditional convergence of random series in Banach spaces. - Lecture Notes in Math., 1980, v. 828, p. 162-166.

11. Kwapien S., Tarieladze $V$. On a.s. unconditional convergence of random series in Banach spaces. - Stochastic Inequalities and Applications (Barcelona, 2002). Basel: Birkhäuser, 2003, p. 71-75. (Progr. Probab., v. 56.)

12. Kwapień S., Szymański B. Some remarks on Gaussian measures in Banach spaces. Probab. Math. Statist., 1980, v. 1, p. 59-65.

13. Gordon Y., Lewis D.R. Absolutely summing operators and local unconditional structures. - Acta Math., 1974, v. 133, p. 27-48.

14. Casazza P. G., Nielsen N. J. A Gaussian average property of Banach spaces. - Illinois J. Math., 1997, v. 41, № 4, p. 559-576.

15. Chobanjan S.A., Tarieladze V.I. Gaussian characterizations of certain Banach spaces. - J. Multivariate Anal., 1977, v. 7, p. 183-203.

16. Johnson W. B. Homogeneous Banach spaces. - Lecture Notes in Math., 1988, v. 1317, p. 201-203.

17. Milman V.D., Pisier G. Banach spaces with a weak cotype 2 property. - Israel J. Math., 1986, v. 54, p. 139-158.

18. Pisier $G$. The volume of convex bodies and Banach space geometry. Cambridge: Cambridge Univ. Press, 1989, 250 p.

19. Talagrand M. Regularity of Gaussian processes. - Acta Math., 1987, v. 159, № 1-2, p. $99-149$.

Поступила в редакцию

31.VIII.2011

Исправленный вариант

1.X.2012 